\section{Sellado marginal de obturaciones de resina compuesta fotoactivadas con luz L.E.D. y luz halógena}

\author{
Marginal seal of composite resin restorations photo \\ activated by L.E.D. and halogen-based light
}

\begin{abstract}
Resumen
El objetivo es comparar in vitro el grado de sellado marginal de restauraciones de resina compuesta fotopolimerizadas con luz L.E.D y con luz halógena convencional. Se emplearon 20 molares sanas recientemente extraídas, en cada una de las cuales se realizaron cavidades clase $V$, una en la superficie vestibular y otra en la superficie palatina de dimensiones estandarizadas. Ambas cavidades fueron obturadas con resina compuesta de nanoparticulas Filteck Z350 y adhesivo Single Bond (3M). Las restauraciones vestibulares se polimerizaron con luz L.E.D., y las palatinas o linguales con luz halógena convencional. Una vez restaurados, los molares se mantuvieron por 48 horas a $37^{\circ} \mathrm{C}$ y $100 \%$ de humedad. Las muestras fueron sometidas a un régimen de termociclaje en una solución de azul de metileno al $1 \%$ para posteriormente evaluar el grado de microfiltración marginal del colorante, realizando un corte en sentido vestíbulo palatino o lingual, y evaluando con un microscopio estereoscopio. Los resultados mostraron que la frecuencia de especímenes que presentaron filtración de colorante fue del $85 \%$ para las obturaciones fotoactivadas con luz convencional y del $50 \%$ para las obturaciones fotoactivadas con luz L.E.D, esta diferencia fue estadísticamente significante $(\mathrm{p}<0.05)$. El grado más severo de penetración de colorante en la interfase diente restauración se presentó en el $60 \%$ de las muestras fotoactivadas con luz halógena y en un $15 \%$ de las muestras fotoactivadas con luz L.E.D, esta diferencia resultó estadísticamente significante, $(\mathrm{p}=0.004)$. Bajo las condiciones del presente estudio se llegó a la conclusión que el grado de sellado marginal de obturaciones de resina compuesta fue significativamente mayor con el fotoactivado de luz L.E.D, que con luz halógena convencional
\end{abstract}

Palabras clave: fotopolimerización, L.E.D, sellado marginal

\section{Abstract}

Objective: to compare in vitro the marginal seal degree of composite resin restorations photopolomerized with L.E.D light and conventional halogen light, was the objective of this research.It was used 20 healthy molars recently extracted, in each of them, standardized dimensions class $\mathrm{V}$ cavities were prepared in the buccal and palatal aspects. Both cavities were filled with nanoparticles composite resin, Filteck Z350 and Single Bond adhesive (3M). Vestibular restaurations were light cured using L.E.D light, and palatal with conventional halogen light. Once restored, the molars were maintained for 48 hours at $37^{\circ} \mathrm{C}$ and $100 \%$ humidity. Samples were subjected to $1 \%$ blue methylene solution thermocycling regimen, and then the marginal leakage degree was assessed, making a palatal-vestibule coronal cut, and the evaluation with a stereoscopic microscopy. The frequency of specimens that leakage the colorant, was $85 \%$ for fillings photoactivated with conventional light, and $50 \%$ for L.E.D, this difference was statistically significant $(\mathrm{p}<0.05)$. The most severe degree of colorant penetration in the tooth-restoration interface was evident in $60 \%$ of samples photoactivated with halogen light, and $15 \%$ with L.E.D, the difference was statistically significant $(p=0.004)$. Under the conditions of this study, it was concluded that marginal seal degree of composite resin fillings was significantly greater with L.E.D light polymerization than conventional halogen light.

Keywords: Photopolymerization. L.E.D. Marginal seal
Artículo Original

\section{Martha Elena Pineda Mejía ${ }^{1}$, Liliana Ángela Terán Casafranca ${ }^{1}$, Waldo Ernesto Gloria Zevallos ${ }^{1}$, Luis Alberto Cuadrao Zavaleta ${ }^{2}$}

1 Dpto. Acad. Estomatología Rehabilitadora. Fac. Odontologia. Universidad Nacional Mayor de San Marcos.

2 Dpto. Acad. Ciencias Básicas. Fac. Odontologia. Universidad Nacional Mayor de San Marcos.

Correspondencia:

Mg. Martha Elena Pineda Mejía

Av. Germán Amézaga s/n, Lima1, Perú Facultad de Odontología. UNMSM

\section{Introducción}

En la década del sesenta aparecieron las resinas compuestas, material que dio buenos resultados a corto plazo y en las pruebas de laboratorio, pero a largo plazo presentaba un alto porcentaje de fallas $^{1,2,3}$, entre otras causas debido a la contracción de polimerización y la consecuente filtración marginal, problema que aún no se ha solucionado y que es la primera causa de fracasos clínicos con este tipo de material restaurador. Sin embargo, cada día el uso de este mate-
Fecha de recepción: 16-06-12 Fecha de aceptación: 20-07-12 rial adquiere mayor preponderancia, ya que se ha mejorado considerablemente sus propiedades fisicoquímicas y estéticas como material rehabilitador.

Para iniciar la reacción química de polimerización se requiere de un aporte de 
energía a través de algún mecanismo de iniciación que puede ser activación química (autocurado), activación por calor (termocurado) y activación por luz visible (fotocurado). ${ }^{4}$ Para este último caso existen varias fuentes lumínicas:

- Lámparas de plasma (de arco, xenón).

- Lámparas de láser.

- Lámparas halógenas: las que pueden ser convencionales (hasta 400$500 \mathrm{~mW} / \mathrm{cm}^{2}$ ) y de alta intensidad o potencia (sobre $500 \mathrm{~mW} / \mathrm{cm}^{2}$ ).

- Lámparas de luz emitida por diodos (L.E.D): con una potencia que va desde los 800 a los $1500 \mathrm{~mW} / \mathrm{cm}^{2}$.

Las lámparas más usadas hasta hoy han sido las lámparas halógenas convencionales.

Recientemente las lámparas de tipo L.E.D. se están incorporando como una alternativa más eficiente según sus fabricantes. Este nuevo sistema de fuente lumínica aparece en el mercado odontológico alrededor del año 1995. Son lámparas que emiten una luz azul con una longitud de onda entre 440-490 nm., con un peak de $460 \mathrm{~nm}^{3}$, permitiendo activar un fotoiniciador que sea sensible a esa longitud de onda y producir la polimerización del material. ${ }^{5}$

El elemento fotoiniciador de la reacción de polimerización más comúnmente utilizado en las resinas compuestas es la Canforoquinona. Esta absorbe energía a un espectro de luz visible entre 400 y $500 \mathrm{~nm}$., con un pico ideal de $468 \mathrm{~nm}$, valor que coincide con el espectro emitido por las lámparas L.E.D., lo que les daría a estas una mayor eficiencia en la fotoactivación. ${ }^{4}$

Por lo tanto, la probabilidad de que un fotón emitido por una lámpara L.E.D. sea absorbido por las Canforoquinonas es considerablemente mayor a que si fuera de una lámpara halógena convencional. ${ }^{4}$ Esto eventualmente se traduciría en que la lámpara L.E.D. lograría producir una fotoactivación de mayor eficiencia y, por lo tanto, una polimerización más completa.

La potencia de las lámparas L.E.D. oscila entre los $800-1400 \mathrm{~mW} / \mathrm{cm}^{2}$, a pesar de que solo se necesita 300-400 $\mathrm{mW} / \mathrm{cm}^{2}$ para lograr una buena polimerización. ${ }^{6}$

Debido a su alta potencia los fabricantes aseguran que, en comparación con una lámpara halógena convencional, las lámparas L.E.D. logran una mayor polimerización de las resinas compuestas con un menor tiempo de exposición lumínica. ${ }^{7}$ Sin embargo, esto podría traducirse en una activación muy brusca, generando tensiones que pueden afectar la calidad del sellado marginal de la restauración.

Por lo cual el propósito del presente estudio es evaluar comparativamente el sellado marginal de restauraciones de resina compuesta fotoactivadas con lámpara L.E.D. con aquellas fotoactivadas con lámpara convencional de luz halógena.

Los objetivos del estudio son:

- Evaluar en una muestra in vitro la frecuencia de casos de filtración marginal de colorante en restauraciones de resina compuesta fotoactivadas con luz L.E.D y con luz halógena convencional.

- Evaluar in vitro el grado de filtración marginal obtenido en las restauraciones de resina compuesta fotoactivadas con luz L.E.D y con luz halógena convencional.

- Analizar comparativamente los resultados obtenidos.

\section{Material y método}

La presente investigación corresponde a un estudio experimental in vitro.

\section{Muestra y su preparación}

Se emplearon 20 piezas dentarias posteriores, molares sanas recientemente extraídas, las cuales después de pasar por un proceso de limpieza fueron mantenidas en una solución de hipoclorito de sodio desde el momento de la exodoncia hasta el momento de iniciar la experimentación. Luego se procedió a la preparación de cavidades clase $\mathrm{V}$, una en la superficie vestibular y otra en la superficie lingual o palatina, con dimensiones estandarizadas $(4 \mathrm{~mm}$ de largo, $3 \mathrm{~mm}$ de ancho y $2 \mathrm{~mm}$ de profundidad), ubicadas a $1 \mathrm{~mm}$ del límite amelocementario (Fig. 1). Las cavidades fueron realizadas usando turbina refrigerada y piedras de diamante cilíndricas de extremo redondeado (ISO n. ${ }^{\circ}$ 14), cambiando la fresa cada 4 cavidades realizadas. Este procedimiento fue realizado por un solo operador entrenado y calibrado.

\section{Material de obturación}

Se usó una resina compuesta de nanorelleno Filteck Z350 (3M/ESPE, St. Paul
MN 55144-1000. Lote 139914. USA) como material de obturación directo en ambas cavidades. Es un material de alta densidad, fotopolimerizable, radiopaco, con indicación para restauración de molares y premolares y como sistema adhesivo se utilizó Single Bond (3M/ ESPE, St. Paul MN 55144, Lote $\mathrm{N}^{\circ}$ 244267.USA).

\section{Sistemas de activación}

Se utilizó una lámpara de luz emitida por diodos L.E.D. GUILIN WOODPECKER MEDICAL INSTRUMENT CO. Ltd. P.R. Guilin, Guangxi, China, con una potencia de $650 \mathrm{~mW} / \mathrm{cm}^{2}$, y una lámpara de luz halógena convencional marca Litex 680 A (Dentamerica Industry, CA 91744 USA), con una potencia de $480 \mathrm{~mW} / \mathrm{cm}^{2}$. (Fig. 2).

\section{Técnica de obturación}

Se procedió a obturar las cavidades con la técnica incremental siguiendo estrictamente las etapas establecidas y sus respectivos tiempos de trabajo, fotoactivando el adhesivo y la resina compuesta con luz L.E.D en las cavidades vestibulares y las palatinas o linguales con luz halogena convencional.

\section{Protocolo de obturación con resina compuesta Z350 (3M)}

Secado preliminar de la pieza dentaria.

Grabado ácido: con ácido ortofosfórico $37 \%$ aplicado con una jeringa, primero solo en esmalte, en los márgenes de la cavidad por $20 \mathrm{~s}$, y luego sobre la totalidad de la cavidad por $10 \mathrm{~s}$ adicionales, de este modo se obtuvo una desmineralización diferencial de esmalte y dentina, ya que se grabó $30 \mathrm{~s}$ en esmalte y $10 \mathrm{~s}$ en dentina.

Lavado y secado: Se lavaron las cavidades con un chorro de agua por 30 s y se procedió a secar la dentina y el esmalte con un chorro de aire, evitando desecar la dentina.

Se aplicó el adhesivo en dos capas consecutivas, la primera frotando la superficie cavitaria con un aplicador microbrush por $15 \mathrm{~s}$, para luego soplar suavemente con un chorro de aire. La segunda capa se aplicó sin frotar y se fotopolimerizó por 20 segundos.

Control de sellado: Se procedió a revisar con el esplorador que el adhesivo cubra todas las paredes dejando una superficie completamente lisa y brillante, si esto no se hubiera logrado, se agregó una nueva capa de adhesivo para con- 
seguir resultados óptimos de sellado dentinario.

Se aplicó el material restaurador en varias capas -técnica incremental- en ambas cavidades operatorias, fotopolimerizando $20 \mathrm{~s}$ cada incremento inicial y por $40 \mathrm{~s}$ el incremento final.

\section{Filtración y termociclado}

Una vez realizadas las restauraciones, se dejaron en la estufa a $37^{\circ} \mathrm{C}$ con un $100 \%$ de humedad, durante 48 horas, luego se sellaron todas las superficies de la pieza dentaria con esmalte para uńas excepto un margen de $2 \mathrm{~mm}$ alrededor de la restauración.

Luego se hizo un proceso de termociclado de las muestras, utilizando una solución acuosa de azul de metileno al $1 \%$ usado como indicador de la microfiltración marginal, el régimen fue de 60 ciclos entre $5^{\circ}$ y $60{ }^{\circ} \mathrm{C}$ manteniendo las muestras 30 segundos en cada baño térmico y llevando a una temperatura ambiente $\left(+/-20^{\circ} \mathrm{C}\right)$ durante $15 \mathrm{~s}$ entre un baño y otro. Continuando las muestras en inmersión pasiva por 72 horas.

Después de este proceso se procedió al lavado y secado de las muestras para posteriormente ser cortadas paralelas al eje mayor del diente pasando por el centro de ambas restauraciones en sentido vestíbulo palatino o lingual, con el fin de analizar la interfase diente-restauración y el nivel de filtración de colorante.

La evaluación de la filtración marginal se hizo en un microscopio estereoscopio con un aumento de (10X).

Se hizo la calificación de los grados de filtración de colorante mediante los siguientes criterios:

$0=$ No hay filtración de colorante.

1 = Filtración en el margen cavitario que abarca la zona de esmalte del diente.

2 = Filtración en el margen cavitario que abarca hasta la zona de dentina del diente.

3 = Filtración en el margen cavitario que abarca la porcion del piso de la cavidad o pared pulpar.

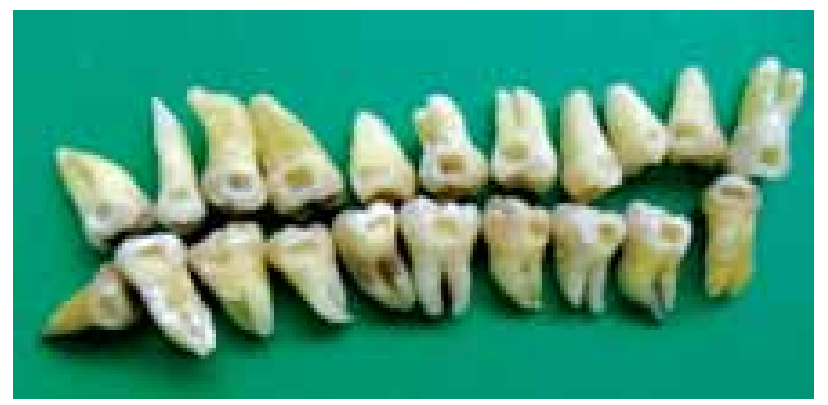

Fig. 1. Molares con preparación de cavidades clase V de dimensiones estandarizadas.

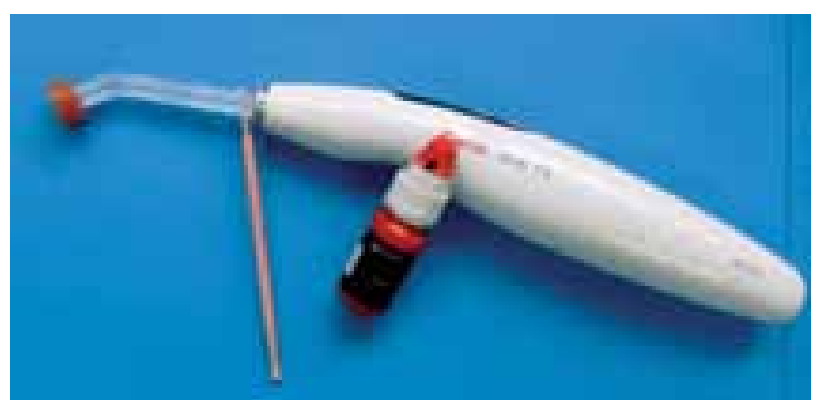

Fig. 2. Lámpra L.E.D.



Fig. 3. Filtración marginal: palatino-luz halógena y vestibular-L.E.D.

\section{Resultados}

La mayor frecuencia de casos con filtración marginal de colorante se presentó en las restauraciones de resina compuesta fotoactivadas con luz halógena convencional.

Tabla1. Distribución de la muestra según la presencia de filtración marginal

\begin{tabular}{lccc}
\hline FILTRACIÓN & No & Sí & Total \\
\hline L. Halógena & $3(15 \%)$ & $17(85 \%)$ & $20(100 \%)$ \\
L. L.E.D. & $10(50 \%)$ & $10(50 \%)$ & $20(100 \%)$ \\
\hline
\end{tabular}

$X^{2}=5.584 p<0.05$

El mayor número de restauraciones de resina compuesta fotoactivadas con luz L.E.D presentaron grado 0 de filtración marginal mientras que el mayor número de restauraciones de resina compuesta fotoactivadas con luz halógena convencional presentaron grado 3 de filtración marginal. (Fig. 3).

Tabla 2. Distribución de la muestra según grados de filtración marginal

\begin{tabular}{lccccc}
\hline GRADOS & 0 & 1 & 2 & 3 & total \\
\hline L. Halógena & $3(15 \%)$ & $3(15 \%)$ & $2(10 \%)$ & $12(60 \%)$ & $20(100 \%)$ \\
L. L.E.D. & $10(50 \%)$ & $3(15 \%)$ & $4(20 \%)$ & $3(15 \%)$ & $20(100 \%)$ \\
\hline
\end{tabular}






$X^{2}=9.8358 p<0.05$

Fig. 4. Presencia de filtración marginal

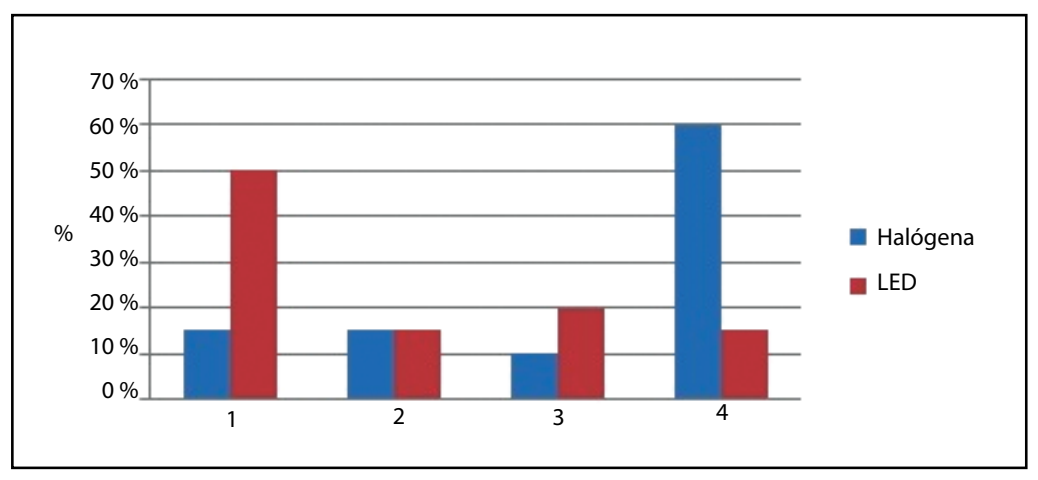

Fig. 5. Grados de filtración marginal en restauraciones de resina compuesta según tipo de fotoactivación

Los datos fueron analizados mediante la prueba chi cuadrado y la prueba $U$ de Mann Whitney-Wilcoxon al $95 \%$ de confianza, dada la naturaleza de las variables en estudio.

\section{Discusión}

La capacidad de una restauración de dar un cierre hermético de la interface con la pieza dentaria es un requisito importante, fundamentalmente debido a la infiltración marginal y su potencial daño a los tejidos y la formación de caries secundarias. Esto es un elemento gravitante al momento de la elección del material restaurador. ${ }^{6}$

La magnitud de la interfase entre el diente y la restauración va a depender tanto de las características propias del material (propiedades físico químicas) como de factores inherentes a la técnica utilizada por el operador y la calidad de los tejidos sobre los cuales se está trabajando. Es por eso que al interpretar los resultados debemos tener en cuenta que en ellos influyen tanto las condiciones experimentales como la técnica utilizada.

$\mathrm{Al}$ analizar los resultados en cuanto a la frecuencia de especímenes que presentaron filtración marginal del colorante, vemos que esta se produjo en un $85 \%$
Esto lo podemos atribuir a que se utilizó una lámpara L.E.D. cuya potencia fue mayor que la lámpara halógena convencional y como ya se estableció, la lámpara L.E.D. concentra toda su potencia en la producción de su espectro de luz azul única a $460 \mathrm{~nm}$; en cambio la lámpara halógena convencional emite un amplio rango de longitud de onda cubriendo una gran parte del espectro, lo cual resulta en la producción de una luz blanca, que gracias a la presencia de un filtro, solo se deja pasar al conductor la luz azul visible que puede oscilar entre los 420-500 nm. ${ }^{8}$

El fotoiniciador de la resina utilizada es un tipo de canforoquinona, esta tiene un pico de fotoactivación a los 468 nm., lo cual es muy cercano al espectro de luz que emite la lámpara L.E.D. ${ }^{10}$ Por lo tanto, como ya se ha señalado, la probabilidad de que un fotón emitido por la lámpara L.E.D. sea absorbido por la canforoquinona es considerablemente mayor a que si fuera de una lámpara halógena convencional. Esto, se traduce en que la luz L.E.D. logra producir una fotoactivación de mayor eficiencia y, por lo tanto, una polimerización más completa y profunda.

Sin embargo, es importante tener en cuenta que fotoiniciadores distintos a la canforoquinona en la resina compuesta; tendrán un pico de absorción diferente al que emite la lámpara L.E.D. produciéndose en todo caso una polimerización deficiente o prácticamente nula, lo que no ocurre con las lámparas halógenas convencionales que abarcan un gran espectro de luz visible, logrando polimerizar incluso aquellas resinas compuestas que utilizan fotoiniciadores distintos a la canforoquinona.

Aunque algunos estudios han señalado que la microfiltración es mayor usando lámpara L.E.D. que lámpara halógena e incluso que una lámpara de plasma ${ }^{8}$. Las microfiltraciones descritas en base a la contracción sufrida por los composites en su proceso de polimerización mediante el uso de emisores de alta intensidad pueden ser obviadas mediante el uso de técnicas incrementales de colocación, actualmente establecidas en la práctica habitual. ${ }^{9}$

Hasta la fecha se han realizado investigaciones independientes que analizan las propiedades mecánicas de las resinas compuestas, como la dureza del material, tensión flexural y módulo de elasticidad, además de la profundidad de polimerización, grado de conversión de los monómeros y filtración marginal ${ }^{10}$ usando la tecnología L.E.D. y 
han demostrado una aparente garantía y seguridad para poder utilizarla en la práctica clínica. ${ }^{11}$

Si bien es cierto que los resultados de esta investigación muestran que las restauraciones fotopolimerizadas con luz L.E.D. presentan una ventaja en términos de filtración marginal respecto a las fotopolimerizadas con luz halógena convencional, no podemos decir que estos resultados sean concluyentes ya que solo se evaluó la filtración del colorante en las paredes oclusal y gingival de cada cavidad, siendo lo ideal medir en todas las superficies de adhesión. ${ }^{12,13}$

\section{Conclusiones}

Bajo las condiciones experimentales de esta investigación y de acuerdo a los resultados obtenidos podemos concluir que, el grado de sellado marginal de obturaciones de resina compuesta fue significativamente mayor con el fotoactivado de luz L.E.D., que con luz halógena convencional

\section{Referencias bibliográficas}

1. Anderson et al. Treating dental caries as an infectious disease Ope-rative dentistry 1991;16:21-28.
2. Craig. Materiales dentales restauradores 7. ${ }^{a}$ ed. Mundi S.A.I.C; 1991. pp: 237-266, pp: 41-65.

3. Philips, R. La ciencia de los materiales dentales. 8. ${ }^{a}$ ed. Interamericana; 1987. pp. 226-260.

4. Jain P, Pershing A: Profundidade de Polimerizacao e micro-infiltracao de resinas compostas com fotopolimerizadores de alta e progressiva intensidade. JADA. 2004; 4(1):51-61

5. Dunn WJ, Bush A C: A comparison of polymerization by light-emitting diode and halogenbased light curing units. JADA. 2002;133:335-341.

6. Yoon H, Lee K, Lim C, Kim W: Degree of polymerization of resin composites by different lights sources: Journal of Oral Rehabilitation. 2002; 29:1165-1173.

7. Sturdevant, C.M., Roberson, T., Heymann, H., Sturdevant, J. Operatoria dental, 3. ${ }^{a}$ ed. Harcourt Brace; 1996. pp: 253-265

8. Hoffmann N, Hugo B, Klaiber B. Effect of irradiation type (LED or QTH) on photo-activated composite shrinkage strain kinetics, tem- perature rise, and hardness. Eur J Oral Sci. 2002 Dec;110(6):471-9.

9. Giner L., Ribera M., Cucurella S, Ferrá J. Lámparas de emisión de diodos (L.E.D.): El futuro de la fotopolimerización. Área de Biomateriales y Prótesis. Universitat Internacional de Catalunya. http:/ www.odontología-online.com/casos/part/MAS/MAS02/mas02.html

10. Harada, K.M.; Caputo,A.A. and Mito, R. Effect of light emitting diode curing on composite resin microleakage. J. Dent. Res. 81 (Sp. Iss A) Abs:485, 2002.

11. Saravia M., Una nueva e innovadora propuesta tecnológica para la fotopolimerización de materiales dentales: Luz emitida por diodos (L.E.D.). 2004. http:/www.odontología-online.com/casos_old/part/ MAS/MAS02/mas02.html

12. Barkmeier W. "Laboratory evaluation of adhesive systems". Operative Dentistry, 1992;5:50-61.

13. Cabanes G.G. Fuentes lumínicas para la fotoactivación en Odontología. Rev Blanq Dent. 2002; E1:17-25 